\title{
Sexually Dimorphic Synaptic Organization of the Medial Amygdala
}

\author{
Bradley M. Cooke and Catherine S. Woolley \\ Department of Neurobiology and Physiology and Institute for Neuroscience, Northwestern University, Evanston, Illinois 60208
}

The medial amygdala is important in social behaviors, many of which differ between males and females. The posterodorsal subnucleus of the medial amygdala (MeApd) is particularly sensitive to gonadal steroid hormones and is a likely site for gonadal hormone regulation of sexually dimorphic social behavior. Here we show that the synaptic organization of the MeApd in the rat is sexually dimorphic and lateralized before puberty. With the use of whole-cell voltage-clamp recording and quantitative electron microscopy, we found that, specifically in the left hemisphere, prepubertal males have $\sim 80 \%$ more excitatory synapses per MeApd neuron than females. In the left but not the right MeApd, miniature EPSC (mEPSC) frequency was significantly greater in males than in females; mEPSC amplitude was not sexually dimorphic. Paired-pulse facilitation of EPSCs, an index of release probability, also was not sexually dimorphic, suggesting that greater mEPSC frequency is caused by a difference in excitatory synapse number. Electron microscopy confirmed that the asymmetric synapse-to-neuron ratio and the total asymmetric synapse number were significantly greater in the left MeApd of males than of females. In contrast to results for excitatory synapses, we found no evidence of sexual dimorphism or laterality in inhibitory synapses. Neither the frequency nor the amplitude of mIPSCs was sexually dimorphic or lateralized. Likewise, the number of symmetric synapses measured with electron microscopy was not sexually dimorphic. These findings show that the excitatory synaptic organization of the left MeApd is sexually differentiated before puberty, which could provide a sexually dimorphic neural substrate for the effects of hormones on adult social behavior.

Key words: amygdala; synapse; rat; sex difference; EPSC; electron microscopy

\section{Introduction}

In the social life of rodents, olfaction is essential. Rodents use their main and accessory olfactory systems for social recognition and territoriality and to initiate neuroendocrine reflexes (Brennan and Keverne, 2004). Male and female rats use olfactory cues differently. For example, males use olfaction to respond to estrous females and to compete for dominance (Bergvall et al., 1991), whereas females depend on olfaction to establish and maintain relations with their litters (Aron, 1979). Consistent with sex differences in sociosexual behavior, the rat olfactory system is sexually dimorphic (Guillamón and Segovia, 1997).

The medial nucleus of the amygdala (MeA) is a key part of the social behavior system of rodents. It receives pheromone cues via the accessory olfactory bulb (AOB) and main olfactory inputs via the cortical nucleus of the amygdala (Scalia and Winans, 1975). MeA lesions produce severe deficits in mating, parental behavior, and social recognition (Harris and Sachs, 1975; Ferguson et al., 2001; Sheehan et al., 2001). In accordance with its role in social

Received July 14, 2005; revised 0ct. 3, 2005; accepted 0ct. 8, 2005.

This work was supported by National Institute of Neurological Disorders and Stroke Grant NS37324, The W. M. Keck Foundation (C.S.W.), and National Institute of Mental Health Grant MH073335 (B.M.C.). We thank Renee May for excellent technical assistance.

Correspondence should be addressed to Bradley M. Cooke, Department of Neurobiology and Physiology, Northwestern University, 2-160 Hogan, 2205 Tech Drive, Evanston, IL 60608. E-mail: b-cooke@northwestern.edu. DOI:10.1523/JNEUROSCI.2919-05.2005

Copyright $\odot 2005$ Society for Neuroscience ～0270-6474/05/2510759-09\$15.00/0 behavior, the MeA is sexually dimorphic. A number of studies have shown structural and neurochemical sex differences in the adult MeA (Nishizuka and Arai, 1981), particularly in the posterodorsal subnucleus (MeApd) (De Vries et al., 1984; Hines et al., 1992; Cooke et al., 1999). Thus, the MeApd is a good model in which to study the regulation of sexually dimorphic brain-behavior relationships.

Understanding how hormones alter neuronal structure and function in adulthood requires an understanding of prepubertal neuronal connectivity. In mammals, sex differences arise through differential exposure to gonadal hormones (Cooke et al., 1998). In males, a perinatal surge of androgen organizes hormone-sensitive brain nuclei to produce a male phenotype; lack of androgen during this period produces a female phenotype. At puberty and during adulthood, changing levels of gonadal hormones act in many of the same parts of the brain to activate sexually dimorphic behaviors.

The volume of the adult MeApd is greater in males than in females (Hines et al., 1992), and this appears to depend, at least in part, on circulating androgen. Castration in adulthood decreases MeApd volume in males, and androgen treatment increases MeApd volume in adult females (Cooke et al., 1999); however, studies on juvenile rough-and-tumble play suggest that the MeA may be sexually dimorphic before puberty. Testosterone implants in the MeA masculinize the frequency of juvenile play (Meaney and McEwen, 1986), whereas bilateral destruction of the amygdala feminizes play frequency (Meaney et al., 1981). 
Thus, adult hormones may act in the MeA on an already sexually differentiated substrate.

The purpose of this study was to determine whether MeApd synaptic organization is sexually dimorphic before puberty. We used whole-cell voltage-clamp recording and quantitative electron microscopy to evaluate synaptic currents and synapse numbers in the prepubertal MeApd. The results indicate that, specifically in the left hemisphere, MeApd neurons in males are contacted by $\sim 80 \%$ more excitatory synapses than in females. These findings show that the prepubertal MeApd is sexually dimorphic and also show a sex difference in synaptic function that corresponds to a difference in synaptic structure.

\section{Materials and Methods}

Animals. Prepubertal male and female Sprague Dawley rats (Harlan, Indianapolis, IN) were housed separately in a $12 \mathrm{~h}$ light/dark cycle with food and water available ad libitum. At 25-29 d of age, animals were anesthetized with Nembutal $(0.1 \mathrm{mg} / \mathrm{kg})$ and killed. Trunk blood was collected from a subset of animals ( 17 males, 11 females) for radioimmunoassay (RIA) analysis of circulating estradiol [17 $\beta$-estradiol doubleantibody RIA kit (Diagnostic Products, Los Angeles, CA); sensitivity, 2.0 $\mathrm{pg} / \mathrm{ml}$ ] and testosterone [testosterone double-antibody RIA kit (ICN Biomedicals, Costa Mesa, CA); sensitivity, $20 \mathrm{pg} / \mathrm{ml}$ ]. All procedures with animals were performed in accordance with the National Institutes of Health Guide for the Care and Use of Laboratory Animals and were approved by the Northwestern University Institutional Animal Care and Use Committee.

Electrophysiology. On each recording day, an assistant to the investigator randomly selected a male or female 25 - to 29-d-old rat and perfused it intracardially with ice-cold, oxygenated artificial CSF (ACSF) containing the following (in mM): $125 \mathrm{NaCl}, 25 \mathrm{NaHCO}_{3}, 25$ dextrose, $2.5 \mathrm{KCl}$, $1.25 \mathrm{NaH}_{2} \mathrm{PO}_{4}, 1 \mathrm{MgCl}_{2}, 2 \mathrm{CaCl}_{2}$; osmolarity, $320 \mathrm{mmol} / \mathrm{kg} ; \mathrm{pH}$ 7.4. The brain was dissected quickly, blocked to contain the MeApd, and bisected sagittally. In counterbalanced order, the left and right MeApds were sliced $(300 \mu \mathrm{m})$ with an oscillating tissue slicer (OTS 4000; Electron Microscopy Sciences, Hatfield, PA). MeApd slices were then incubated in warm $\left(35^{\circ} \mathrm{C}\right)$, oxygenated ACSF for $40 \mathrm{~min}$. After this, slices were kept in oxygenated ACSF at room temperature for no more than $3 \mathrm{~h}$ after dissection. To begin recording, a slice containing the right or left MeApd was moved to a recording chamber perfused with warm $\left(33^{\circ} \mathrm{C}\right)$, oxygenated ACSF at a rate of $5 \mathrm{ml} / \mathrm{min}$.

An Axioskop microscope (Zeiss, Gottingen, Germany) equipped with infrared filters, differential interference-contrast optics, a video camera (Hamamatsu Photonics, Hamamatsu City, Japan), and a 40× (numerical aperture $=0.8$ ) water-immersion lens was used to visualize and target MeApd neurons. Whole-cell voltage-clamp recordings of synaptic currents were made with glass electrodes (A-M Systems, Carlsborg, WA; outer diameter, $1.5 \mathrm{~mm}$; inner diameter, $0.86 \mathrm{~mm}$ ) pulled to a resistance of 3-5 M $\Omega$. Spontaneous EPSCs (sEPSCs) and synaptically evoked EP$\mathrm{SCs}$ were recorded with a K-gluconate-based internal solution containing the following (in mM): $115 \mathrm{~K}$-gluconate, $20 \mathrm{KCl}, 10 \mathrm{Na}_{2} \mathrm{PG}, 10$ HEPES, 2 EGTA, 2 MgATP, 0.3 NaGTP; osmolarity, 314 mmol/kg; pH 7.3. External ACSF contained the $\mathrm{GABA}_{\mathrm{A}}$ receptor antagonist SR 95531 (GABAzine, $2 \mu \mathrm{M}$; Tocris, Ellisville, MO). sIPSCs were recorded with a CsCl-based internal solution containing the following (in mM): $140 \mathrm{CsCl}$, $2 \mathrm{MgCl}, 10 \mathrm{Na}_{2}$ PG, 10 HEPES, 2 EGTA, 2 MgATP, $0.3 \mathrm{NaGTP}$; osmolarity, $300 \mathrm{mmol} / \mathrm{kg}$; $\mathrm{pH}$ 7.3. External ACSF contained the glutamate receptor antagonist kynurenic acid (2 mm; Sigma, St. Louis, MO). Miniature EPSCs (mEPSCs) or mIPSCs were recorded in ACSF containing the appropriate receptor antagonist plus tetrodotoxin (TTX, $1 \mu \mathrm{M}$; Tocris). Both intracellular solutions contained biocytin $(0.1 \%)$ to visualize recorded cells and $500 \mu \mathrm{M}$ QX-314 (Tocris) to block sodium currents. Signals were amplified and filtered with an Axopatch 200B amplifier (2 kHz; Molecular Devices, Foster City, CA), digitized with an ITC-18 I/O 18-bit board (Instrutech, Port Washington, NY), and stored on the hard drive of a Macintosh G4 computer. IgorPro 3.0 software (Wavemetrics, Lake Oswego, OR) running custom-built data acquisition procedures (DataPro 4.0; courtesy of Nelson Spruston, Northwestern University) was used to create paired-pulse stimulation protocols. Paired pulses were generated by a stimulus isolation unit (AMPI, Jerusalem, Israel) and delivered via a bipolar theta glass electrode filled with ACSF that was placed $\sim 100 \mu \mathrm{m}$ from the recorded neuron. Half-maximal paired pulses were delivered at $0.1 \mathrm{~Hz}$, with at least three sweeps per interstimulus interval. An experiment was discarded if series resistance exceeded 20 $\mathrm{M} \Omega$. The holding potential in all experiments was $-70 \mathrm{mV}$.

Data analysis was conducted off-line with IgorPro 3.0 running Neuromatic procedures (Jason Rothman, http://www.neuromatic.thinkrandom.com/). Spontaneous and miniature postsynaptic currents were analyzed with the "Event" procedure of the Neuromatic software. At least 500 events were recorded in each spontaneous and miniature condition, and each detected event was inspected visually before inclusion in the comparison of EPSC and IPSC frequencies and amplitudes.

Electron microscopy. Male $(n=4)$ and female $(n=5) 25$ - to 27 -d-old rats were gravity perfused with heparinized $0.9 \% \mathrm{NaCl}$ followed by cold $2 \%$ paraformaldehyde $/ 2 \%$ glutaraldehyde in $0.1 \mathrm{~m}$ phosphate buffer, $\mathrm{pH}$ 7.4 , for $20 \mathrm{~min}$. The brain was removed, and the right cerebral cortex was scored. Brains were then coded and postfixed overnight at $4^{\circ} \mathrm{C}$. The next day, the MeApd was sectioned $(60 \mu \mathrm{m})$ through its rostrocaudal extent with an oscillating tissue slicer. The slices were fixed with osmium, dehydrated in an ascending series of alcohols, and flat embedded in Eponate resin (Electron Microscopy Sciences). From each brain, three blocks were systematic-randomly selected; the MeApd was dissected from them and mounted on BEEM capsules (Structure Probe, West Chester, PA). From each of the three MeApd blocks, a single $1 \mu \mathrm{m}$ semithin section was used to systematic-randomly select four areas for imaging. Then, 10-12 ultrathin serial sections $(\sim 70 \mathrm{~nm})$ were cut on a Reichert Ultracut $S$ ultramicrotome (Leica, Bannockburn, IL), mounted on Formvar-coated slot grids, and stained with uranyl acetate and Reynold's lead citrate. The four systematic-randomly selected areas were then imaged through the series at 20,000 $\times$ with a JEOL 1230 electron microscope (JEOL, Peabody, MA) equipped with a CCD camera. To estimate neuron density, 10-12 semithin serial sections $(1 \mu \mathrm{m})$ were cut from the same systematicrandomly selected MeApds, mounted on slides, Nissl stained, and coverslipped.

An additional set of ultrathin sections was cut to evaluate the morphology of GABA-immunoreactive presynaptic boutons. Periodic acidetched sections were incubated in rabbit anti-GABA IgG (1:500; Sigma) for $3 \mathrm{~h}$, followed by goat anti-rabbit IgG conjugated to $20 \mathrm{~nm}$ colloidal gold particles (1:20; Ted Pella, Redding, CA) for $1 \mathrm{~h}$ at room temperature. Sections were counterstained with uranyl acetate and Reynold's lead citrate and visualized with electron microscopy.

Stereological analyses. Synapses were counted in each stack of 10-12 images with the physical disector (Mouton, 2002). To improve sampling efficiency, the disector (counting frame area, $35 \mu \mathrm{m}^{2}$ ) was applied to each stack two times; synapses were counted and classified in ascending and again in descending serial order, and the counts were averaged for each stack. A synapse was identified by the apposition of a postsynaptic density with a bouton containing at least three vesicles within $50 \mathrm{~nm}$ of the presynaptic membrane. Each synapse was classified as located on a dendritic shaft, a dendritic spine, or a soma and as being asymmetric or symmetric. Asymmetric and symmetric synapses are distinguished by the relative abundance of electron-dense material on the presynaptic and postsynaptic sides of the synapse. Asymmetric synapses contain more electron-dense material on the postsynaptic side (Peters et al., 1991). Too few axosomatic synapses were present in our sample for quantitative analysis. Synapse density was calculated within each MeApd by dividing the mean number of synapses from the two counts by the sampled volume. An average of $142( \pm 7.2)$ synapses were counted in each animal. This sampling scheme resulted in coefficient of error values of 0.06 and 0.01 for the male and female synapse density estimates, respectively.

The number of neurons in the MeApd was estimated to calculate synapse-to-neuron ratios. First, the regional volume of the MeApd was estimated in four male and four female 25-d-old Sprague Dawley rats perfused with $4 \%$ paraformaldehyde. Separate animals were used for this procedure because the boundaries of the MeApd could not be reliably determined in semithin sections of osmium-stained tissue. The brains of these animals were coded, serially sectioned $(40 \mu \mathrm{m})$ through the MeA, 
and stained with cresyl violet. An investigator then traced the MeApd with a camera lucida. Total cross-sectional area was then multiplied by section thickness to estimate MeApd volume.

MeApd neuronal density was estimated by using stereology in the cresyl violet-stained $40 \mu \mathrm{m}$ sections (optical disector) and in the semithin serial sections cut from the electron microscopy material (physical disector). In these analyses, both the MeApd-containing sections and the regions within them were selected systematic-randomly. Neurons were visualized with an Olympus BX60 microscope (Olympus America, Melville, NY) equipped with a $50 \times$ oil objective (numerical aperture, 0.9 ) and a DC330 color video camera (Dage-MTI, Michigan City, MI) and were identified as cells with a large clear nucleus and distinct nucleolus. The counting frame area was $1600 \mu \mathrm{m}^{2}$ in each analysis. The average number of neurons counted per animal was $97( \pm 9.3)$ for the semithin sections and $281( \pm 13.3)$ for the $40-\mu$ m-thick sections. Neuron density estimates from the two sets of tissue differed from each other by $<7 \%$, indicating similar tissue shrinkage with each fixation procedure. Each rat's neuron density, estimated from the semithin sections, was multiplied by the mean regional MeApd volume of that rat's sex to estimate the total number of MeApd neurons. Likewise, the density of each synapse type within each rat's MeApd was multiplied by the mean volume of the MeApd for that rat's sex to estimate the total number of synapses. Finally, the total number of each synapse type was divided by the total number of neurons in the MeApd to estimate synapse-toneuron ratios for each rat.

MeApd soma sizes were measured because this parameter is sexually dimorphic in adult animals and may reflect levels of circulating androgen (Cooke et al., 1999). Forty neurons per animal were randomly selected during the analysis of cell density in the $40-\mu \mathrm{m}$-thick sections. The outline of each cell was traced with NIH Image, and the mean cross-sectional area of traced somata was then used in the comparison of soma size.

Statistics. Results are expressed as means \pm SEM. Electrophysiological data were initially analyzed with two-way, repeated-measures ANOVA, with sex and cerebral hemisphere as independent variables. Only if main effects or significant interactions were detected were Tukey's post hoc tests used to examine effects within or between hemispheres, with $n$ as the number of cells. Neuroanatomical data were analyzed with unpaired, two-tailed Student's $t$ tests, with $n$ as the number of rats. In both sets of analyses, significance was set as $\alpha=0.05$.

\section{Results}

Functional sex differences in the MeApd

Our electrophysiological experiments were designed to determine whether the functional organization of the prepubertal MeApd is sexually dimorphic. We evaluated both excitatory and inhibitory synaptic physiology in MeApd slices from 25- to 29-dold male and female rats. Previous reports that the MeA is lateralized (Gerendai and Halász, 1997; Cooke et al., 2003) led us to examine the left and right MeApd slices separately.

\section{Circulating gonadal hormone levels were low and not sexually dimorphic}

First, to verify that the animals in our study were prepubertal, we assayed the concentrations of testosterone and $17 \beta$-estradiol in the trunk blood of a subset of animals that were perfused for electrophysiology. RIAs confirmed that the levels of these hormones were several times lower than those reported for adult animals (Baulieu and Kelly, 1990; Smith et al., 1992) and were not sexually dimorphic. The results for testosterone were $67 \pm 24$ $\mathrm{pg} / \mathrm{ml}$ for males $(n=13)$ and $28 \pm 28$ for females $(n=11)$; the results for estradiol were $4.7 \pm 1.0 \mathrm{pg} / \mathrm{ml}$ for males $(n=17)$ and $5.3 \pm 0.9 \mathrm{pg} / \mathrm{ml}$ for females $(n=11)$. For both $t$ tests, $p \geq 0.3$.

\section{EPSCs are sexually dimorphic and lateralized}

We recorded sEPSCs in ACSF plus GABAzine in 10-13 MeApd neurons from each hemisphere of 23 males and 21 females. A
A
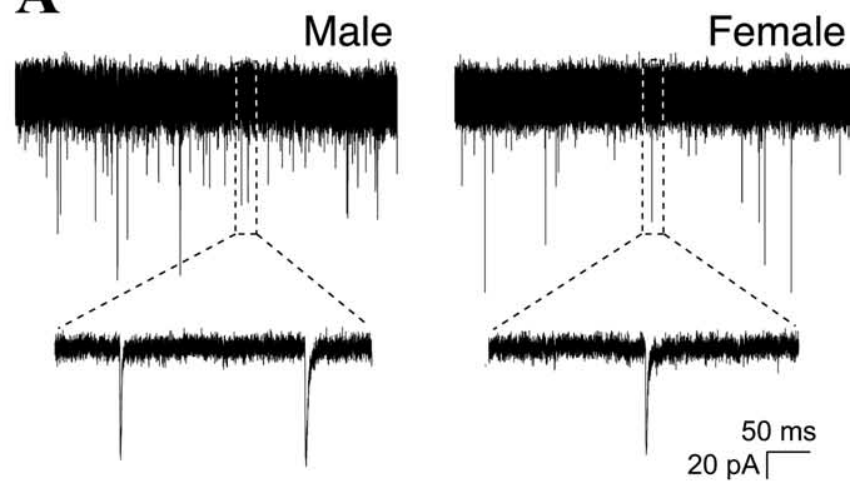

B

B LEFT

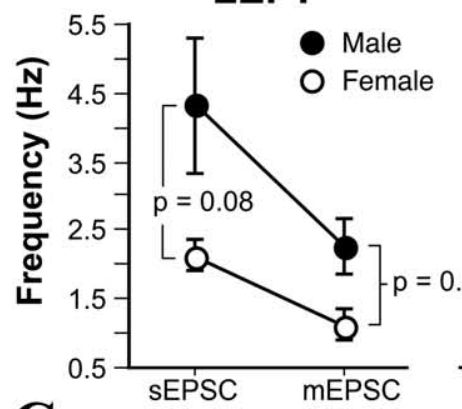

RIGHT

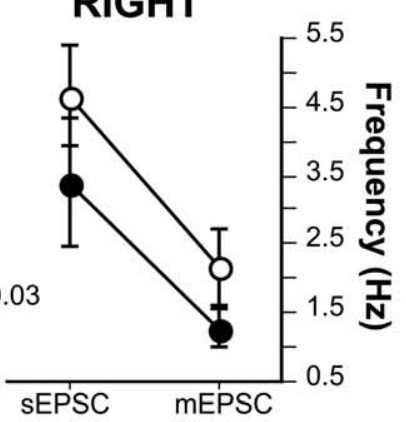

C
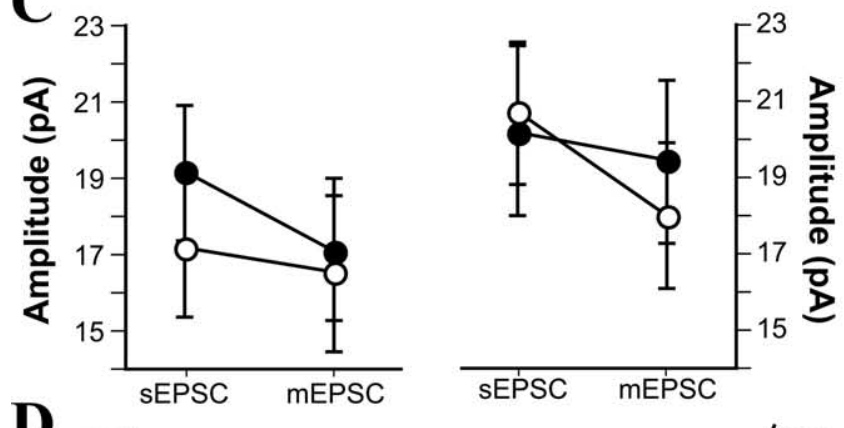

D

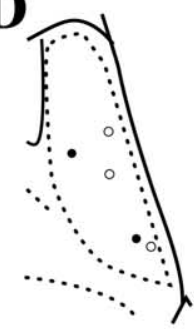

CAUDAL
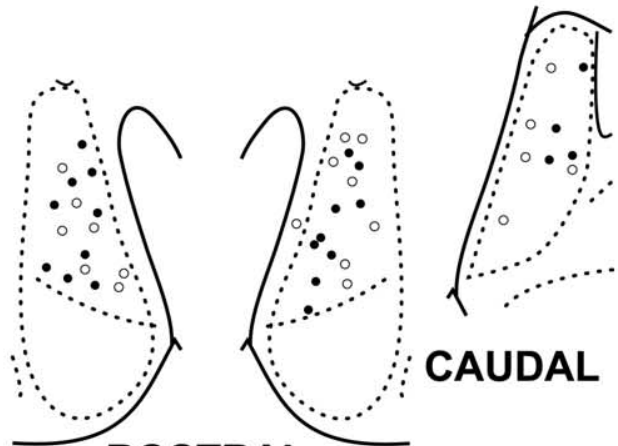

\section{ROSTRAL}

Figure 1. Sex difference in left hemisphere mEPSC frequency. $A$, Representative examples of $\mathrm{mEPSC}$ recorded from male and female MeApd neurons. $\boldsymbol{B}$, Mean sEPSC and mEPSC frequencies recorded from male and female, left and right MeApd neurons. Male neurons had more frequent mEPSCs than females in the left, but not the right, hemisphere. $C$, Mean sEPSC and mEPSC amplitudes recorded from male and female, left and right MeApd neurons. No effect of sex or hemisphere was detected. $\boldsymbol{D}$, Plot of male $(\mathbf{O})$ and female $(\bigcirc)$ cell positions in the left and right MeApd.

total of 49 cells were recorded from 44 animals. After this, TTX was added to the bath to record mEPSCs in each cell (Fig. 1A). sEPSCs represent both action potential-driven synaptic currents and miniature synaptic currents, which reflect the spontaneous 
release of glutamate from individual synaptic sites. The frequency of mEPSCs is a functional index of the number of glutamate release sites per cell, and the amplitude of mEPSCs is a measure of the strength of each site (Hsia et al., 1998; Regehr and Stevens, 2001).

We analyzed at least 500 sEPSCs and 500 mEPSCs per cell. These analyses showed that the frequency of both sEPSCs and mEPSCs in the left hemisphere is more than twice as high in males (Fig. $1 B$ ). Although the difference in sEPSC frequency represented only a statistical trend (Tukey's post hoc test; $p=0.08$ ), the blocking of action potentials with TTX revealed a significant sex difference in mEPSC frequency. In males, mEPSC frequency was $2.26 \pm 0.4 \mathrm{~Hz}$, whereas in females, mEPSC frequency was only $1.12 \pm 0.2 \mathrm{~Hz}$ (Tukey's post hoc test; $p=0.03$ ) (Fig. $1 B$ ). In contrast to results for the left MeApd, no differences or trends toward differences in sEPSC or mEPSC frequency were observed for neurons from the right MeApd (Fig. $1 B$ ). In females, sEPSC frequency was greater on the right side than the left (Tukey's post hoc test; $p=0.003$ ), with values similar to males in both hemispheres; males showed no laterality in either sEPSC or mEPSC frequency. In contrast to sEPSC and mEPSC frequency, comparison of EPSC amplitudes revealed no sex or hemispheric differences or trends (Fig. 1C). The lack of sex difference in sEPSC and mEPSC amplitudes suggests that the strength of MeApd excitatory synapses is not sexually dimorphic.

The pipettes used for electrophysiological recording contained biocytin to visualize neurons after recording. To confirm that there was no sampling bias in the locations of recorded cells, we plotted the locations of biocytin-filled somata on a standard atlas (Fig. 1D). The distribution of recorded cells was not sex biased. Additionally, the fact that cells were well distributed within the MeApd indicates that the sex difference in mEPSC frequency on the left side is likely to be a property of the entire left MeApd, rather than a subset of its cells.

Two not mutually exclusive synaptic differences in the MeApd could underlie greater mEPSC frequency in males: a greater number of excitatory synapses per neuron and/or greater probability of release. One way to evaluate release probability is with paired-pulse experiments in which two synaptic stimuli are given with a brief interstimulus interval. The amplitude of the EPSC evoked by the second pulse is compared with that evoked by the first to evaluate synaptic facilitation (second response is larger) or depression (second response is smaller). Particularly in the case of paired-pulse facilitation, the degree of facilitation is inversely related to the probability of release (Zucker, 1989; Dobrunz and Stevens, 1997).

To evaluate paired-pulse facilitation-depression at excitatory synapses in the MeApd, we applied synaptic stimuli to 14 male and 16 female neurons, which were obtained from 14 male and 13 female rats. Interstimulus intervals ranged from 10 to $250 \mathrm{~ms}$ (Fig. 2A). With these intervals, MeApd synapses in both males and females were exclusively facilitating. Plots of the paired-pulse ratio for male and female neurons in both the left and right hemispheres revealed no sex differences or laterality in paired-pulse facilitation (Fig. $2 \mathrm{~B}$ ). These results indicate that the higher probability of release is not an underlying cause of greater mEPSC frequency in males and point to the possibility of a structural sex difference with more excitatory synapses in the left MeApd of males.

\section{IPSCs are neither sexually dimorphic nor lateralized}

Next we asked whether IPSCs in the MeApd are sexually dimorphic and/or lateralized, as EPSCs are. We recorded sIPSCs in

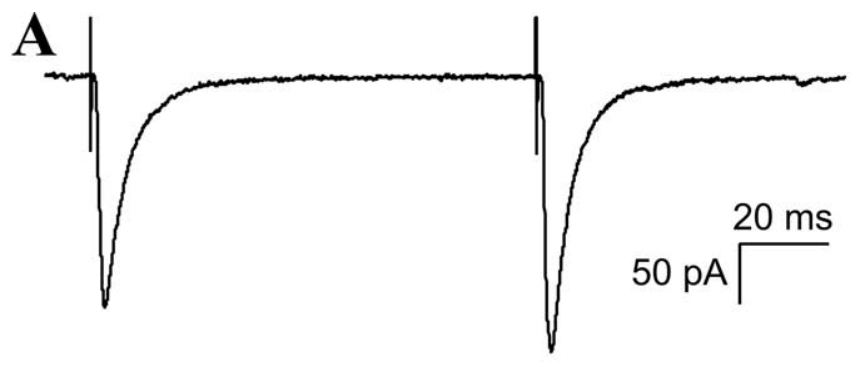

B

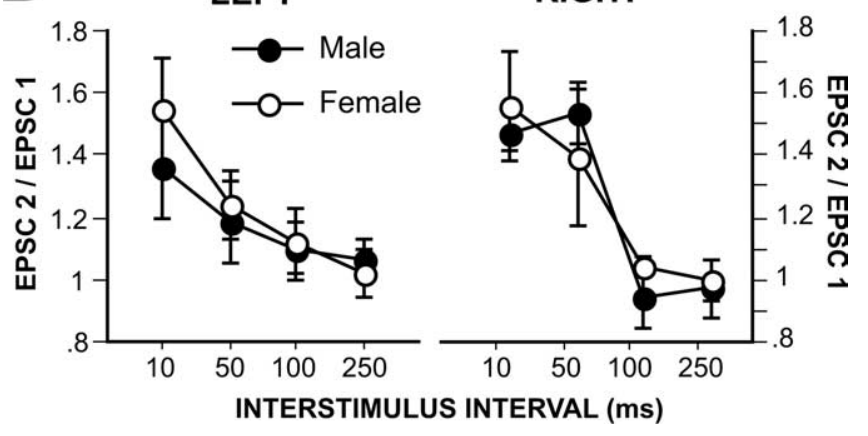

Figure 2. $\quad \boldsymbol{A}$, Example of evoked EPSCs recorded from an MeApd neuron. $\boldsymbol{B}$, Paired-pulse ratios plotted for male and female, left and right MeApd neurons. No effect of sex or hemisphere was detected in paired-pulse ratios.

ACSF containing kynurenic acid to block excitatory neurotransmission, followed by the addition of TTX to record mIPSCs (Fig. $3 A$ ), in 9-15 MeApd neurons from each hemisphere of 17 males and 12 females. A total of 38 cells were recorded from 29 animals. A comparison of sIPSCs and mIPSCs revealed no sex differences or laterality in either event frequency (Fig. 3B) or amplitude (Fig. $3 C)$. Similar to the analysis of EPSCs, we labeled recorded neurons with biocytin and plotted their location within the MeApd. An evaluation of the locations of recorded cells showed no evidence of a sex bias in the distribution of somata (Fig. 3D). Together, these data indicate that, unlike excitatory synapses, neither the number nor the strength of inhibitory synapses in the MeApd is sexually dimorphic.

\section{Structural sex differences in the MeApd}

The lack of a sex difference in the paired-pulse ratio for EPSCs suggested that greater mEPSC frequency in the left MeApd of males could be caused by a greater number of excitatory synapses. We evaluated this possibility with electron microscopy and the physical disector to estimate synapse density, synapse number, and synapse-to-neuron ratio in the left MeApd of four male and five female 25- to 27-d-old rats. The principal findings of this analysis were a greater overall number of excitatory synapses in males and a greater excitatory synapse-to-neuron ratio (see below).

Before counting synapses, we used postembedding immunogold labeling for GABA to confirm that we could identify excitatory and inhibitory synapses by morphological criteria. An examination of GABA-labeled sections (Fig. 4) showed that gold particles were associated with axonal boutons apposed to symmetric synaptic profiles, whereas boutons associated with asymmetric synapses consistently lacked gold particles. This validated the use of symmetric presynaptic and postsynaptic densities as morphological characteristics of GABAergic synapses in the MeApd, as is true elsewhere in the brain (Peters et al., 1991). Most 


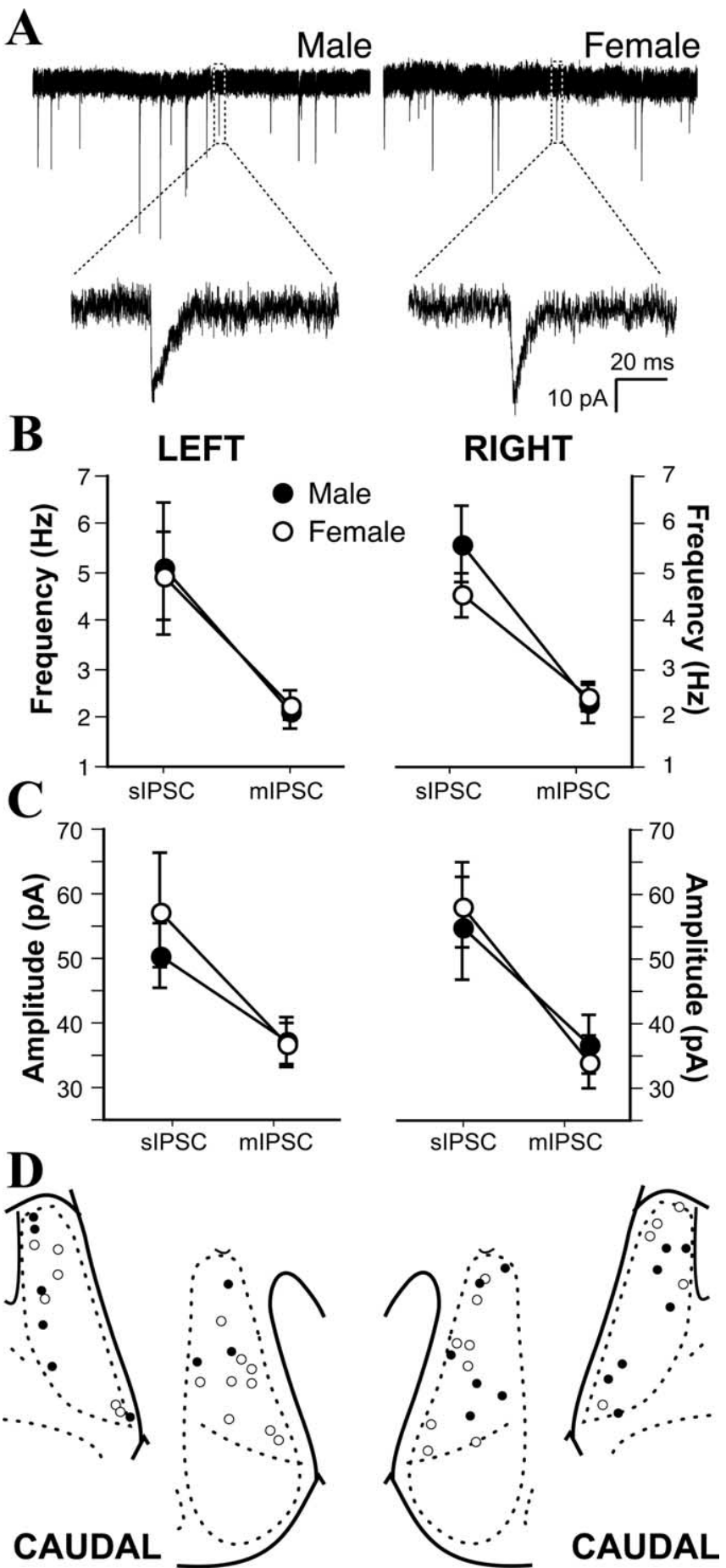

ROSTRAL

Figure 3. No sex differences in inhibitory synaptic physiology. $\boldsymbol{A}$, Representative examples of mIPSCs recorded from a male and a female MeApd neuron. $\boldsymbol{B}$, No sex differences or effects of laterality in sIPSC and mIPSC frequencies. $C$, No sex differences or effects of laterality in sIPSC or mIPSC amplitudes. D, Plot of male $(\mathbf{O})$ and female $(\bigcirc)$ cell positions in the left and right MeApd.

symmetric GABA-labeled synapses were observed on dendritic shafts, but they also appeared on dendritic spines, including on spine heads (Fig. 4, inset). When a spine with a GABA-labeled symmetric synapse was fully contained within the series of sections, an asymmetric synapse was invariably also found on the spine head.

The left MeApd is larger in male rats, with no sex difference in neuron number

Calculation of the synapse-to-neuron ratio depends on the estimation of regional volume, neuron density, and neuron number. These analyses revealed a sex difference in the volume of the left prepubertal MeApd. We measured MeApd volume from thick $(40 \mu \mathrm{m})$ serial sections (Fig. $5 A$ ) and found that it was $18 \%$ greater in males than in females $\left(t_{(6)}=2.55 ; p<0.05\right)$. Specifically, the average regional volume in males was $0.234 \pm 0.010$ $\mathrm{mm}^{3}$; in females, the average regional volume was $0.193 \pm 0.009$ $\mathrm{mm}^{3}$. This difference in prepubertal animals is smaller than the $50 \%$ volume difference observed between gonadally intact adult males and females (Hines et al., 1992; Cooke et al., 1999), suggesting that the additive effects of perinatal and adult androgens produce the sex difference in MeApd volume that is evident in adulthood.

We next measured MeApd neuron density, both with the optical disector in the same thick sections used for regional volume analysis and with the physical disector in the series of semithin (1 $\mu \mathrm{m}$ ) sections (Fig. $5 B$ ) of osmium-stained tissue used for electron microscopy. Both of these analyses showed that neuron density is not statistically different between males and females, although mean values were higher in females. In the osmium-stained tissue, male cell density was $1.0 \pm 0.12 \times 10^{-5} \mathrm{cells} / \mathrm{mm}^{3}$, and female cell density was $1.5 \pm 0.22 \times 10^{-5}$ cells $/ \mathrm{mm}^{3}\left(t_{(7)}=1.92\right.$; $p=0.09$ ). Estimates of neuron density made from thick and semithin sections were similar: they differed by $<7 \%$.

Finally, we calculated total MeApd neuron number by multiplying neuron density by regional volume. This analysis revealed no significant difference between males and females $\left(t_{(7)}=1.11\right.$; $p=0.3$ ); slightly greater neuron density in females compensated for the greater regional volume in males. Male neuron number was 24,196 \pm 2933 ; female neuron number was 30,484 \pm 4435 . Additionally, the mean soma size of MeApd neurons was not different between males and females (males, $95.58 \pm 7.7 \mu \mathrm{m}^{2}$; females, $81.36 \pm 11 \mu \mathrm{m}^{2} ; p=0.2$ ). Together, these results suggest that a component of the MeApd other than the number of neurons or soma size must account for the volumetric sex difference. For example, MeApd neurons in males may have larger dendritic trees, or the male MeApd may contain more or larger glia than the female.

\section{Number of excitatory synapses in the left MeApd is sexually dimorphic}

We used the physical disector method to count synapses in the left MeApd of male and female rats. Each synapse was classified as located on a dendritic spine or shaft and as asymmetric or symmetric. The synapse-to-neuron ratio was calculated by dividing the estimated number of each synapse type per animal by the estimated number of neurons. Consistent with the greater mEPSC frequency in males, electron microscopic analysis of asymmetric synapses on dendritic spines (Fig. 6A) and shafts (Fig. $6 B$ ) revealed that males have a significantly higher synapseto-neuron ratio than females $\left(t_{(7)}=2.49 ; p=0.04\right.$ ) (Fig. $6 C$ ). On average, each MeApd neuron in males had 33,348 \pm 6600 asymmetric synapses, whereas the synapse-to-neuron ratio in females was only $17,814 \pm 1900$. The relative difference was similar for asymmetric spine and shaft synapses, although only asymmetric spine synapses reached statistical significance; asymmetric spine synapses were twofold more numerous on male neurons than on female neurons $\left(t_{(7)}=2.88 ; p=0.02\right)$, and asymmetric shaft synapses were 1.8 -fold more numerous $\left(t_{(7)}=1.77 ; p=0.1\right)$. No 
asymmetric synapses on neuronal somata were observed in our sample. There was a trend toward greater asymmetric synapse density in males $\left(t_{(7)}=1.50 ; p=0.07\right)$ (Fig. $6 D$ ). Together with the greater volume of MeApd neurons in males, this resulted in a total asymmetric synapse number that was significantly greater in males $\left(t_{(7)}=2.91 ; p=0.02\right.$ ) (Fig. $6 E$ ). Because asymmetric synapses were negative for GABA in the immunolabeling analysis, they are likely to be excitatory. Thus, the functional sex difference in mEPSC frequency is associated with a structural sex difference (i.e., more excitatory synapses per neuron in males).

\section{Number of inhibitory synapses in the left MeApd is not sexually dimorphic} In contrast to results for asymmetric synapses, none of the measures related to symmetric synapses were sexually dimorphic. An analysis of symmetric synapses on dendritic spines (Fig. 7A) and shafts (Fig. $7 B$ ) revealed equal synapse-to-neuron ratios in males and females (Fig. 7C). Furthermore, the density of symmetric spine and shaft synapses (Fig. 7D) and their overall number (Fig. 7E) were approximately equal between males and females. These results are consistent with the electrophysiology data, in which we found no sex differences in sIPSC or mIPSC frequency. Interestingly, the estimated total number of symmetric synapses in the MeApd was $\sim 1.5$ times lower than the number of asymmetric synapses (compare Figs. $6 D, 7 D)$.

\section{Discussion}

We have shown that the prepubertal MeApd is sexually dimorphic at the functional and structural levels. In the left MeApd, mEPSC frequency, regional volume, and asymmetric synapse-to-neuron ratio were all greater in males than in females. The levels of circulating testosterone and estradiol were low and not sexually dimorphic at this age, which indicates that these sex differences are not caused by the activational effects of gonadal steroids. Instead, the data suggest that perinatal exposure to androgens or their metabolites affects the number of excitatory connections. In contrast, there was no evidence that inhibitory synapses in the MeApd are sexually dimorphic at either the functional or the structural level. To our knowledge, this is the first report that shows a correspondence between differences in synaptic physiology and synaptic structure in a sexually dimorphic brain nucleus.

Our results provide new insight into MeA sex differences in adulthood. Nishizuka and Arai (1981) previously reported greater areal density of dendritic shaft synapses in the adult male synapse. used to identify neurons.

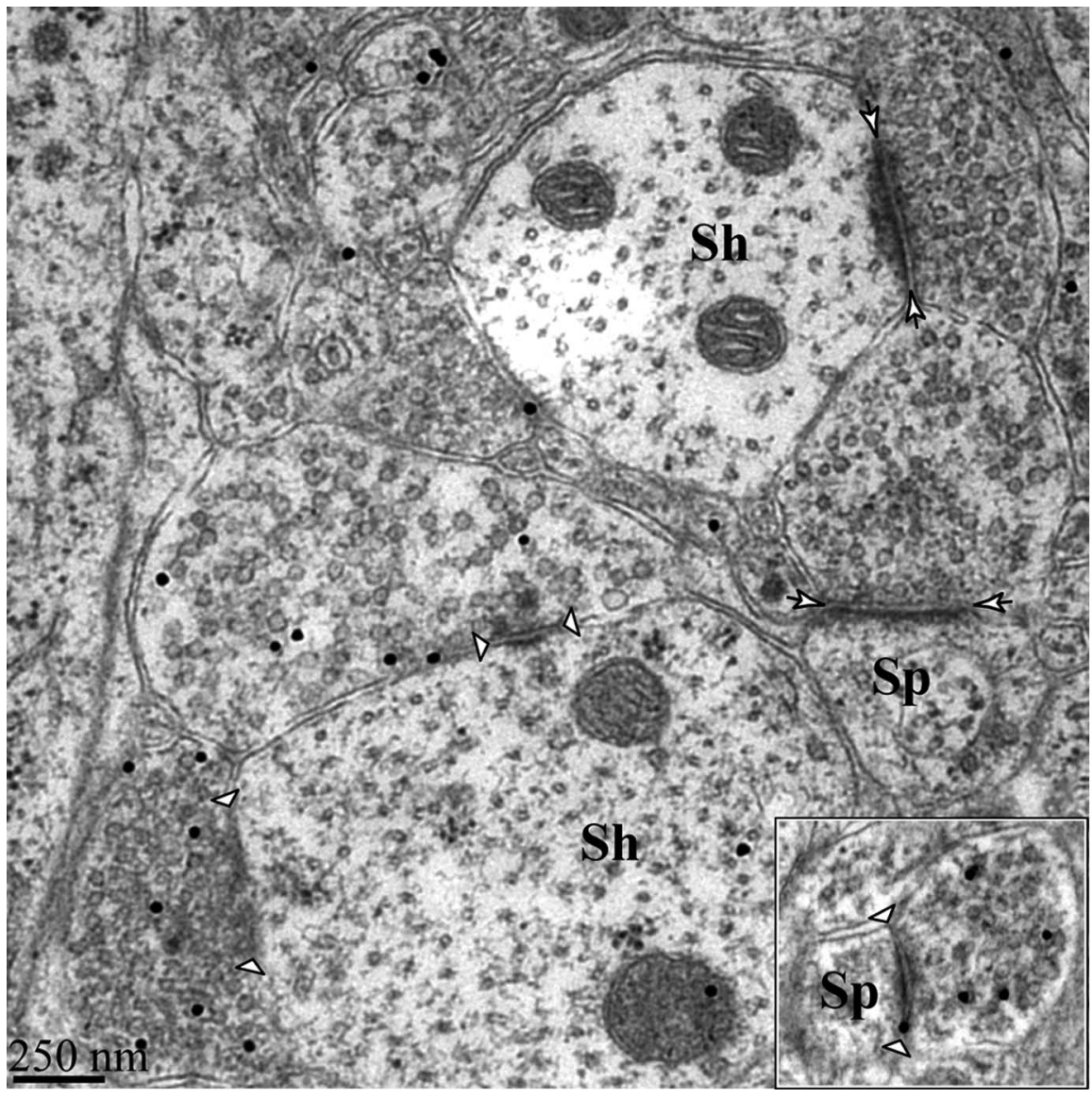

Figure 4. Symmetric synapses are immunoreactive for GABA. Shown are examples of symmetric dendritic shaft synapses (indicated by arrowheads) immunopositive for GABA and asymmetric dendritic spine and shaft synapses immunonegative for GABA (indicated by short arrows). Inset, Example of immunopositive symmetric spine synapse. Sh, Shaft synapse; Sp, spine
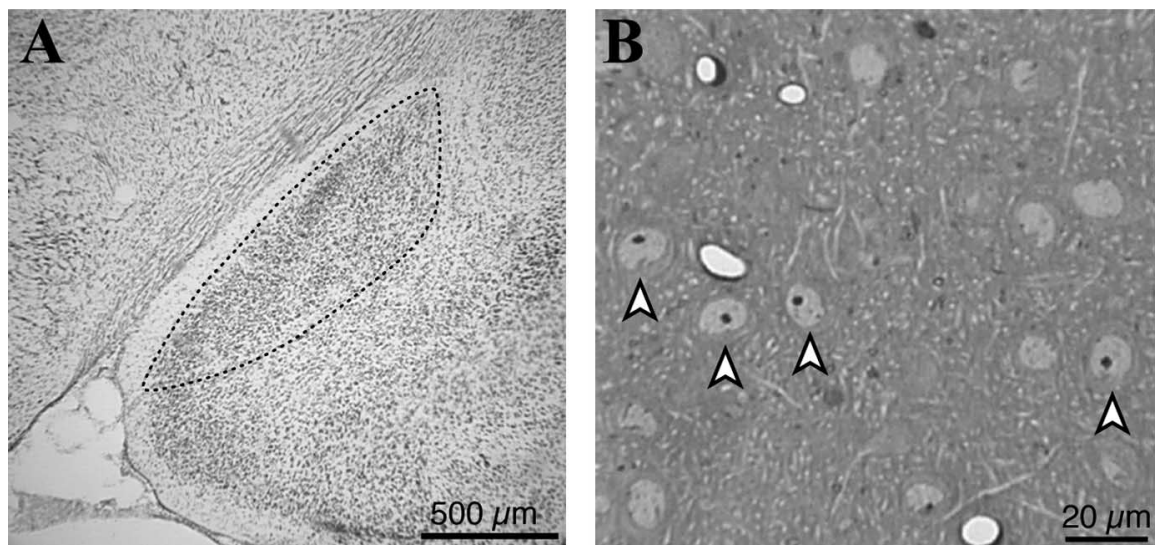

Figure 5. Light microscopic analysis of MeApd morphology. $\boldsymbol{A}$, Photomicrograph of the left MeApd in a male brain section stained with cresyl violet. $\boldsymbol{B}$, Photomicrograph of a semithin-sectioned MeApd illustrates the characteristic nuclei (arrowheads)

MeA. This study did not distinguish between symmetric and asymmetric synapses, however, so it was unknown whether additional synapses in males were excitatory or inhibitory. Our findings indicate that this sex difference is likely to be in excitatory synapses and, furthermore, that excitatory synaptic connectivity is sexually differentiated before puberty. In addition, we found that the sex difference in MeApd volume in adulthood (Hines et al., 1992) is also present before puberty, although to a 

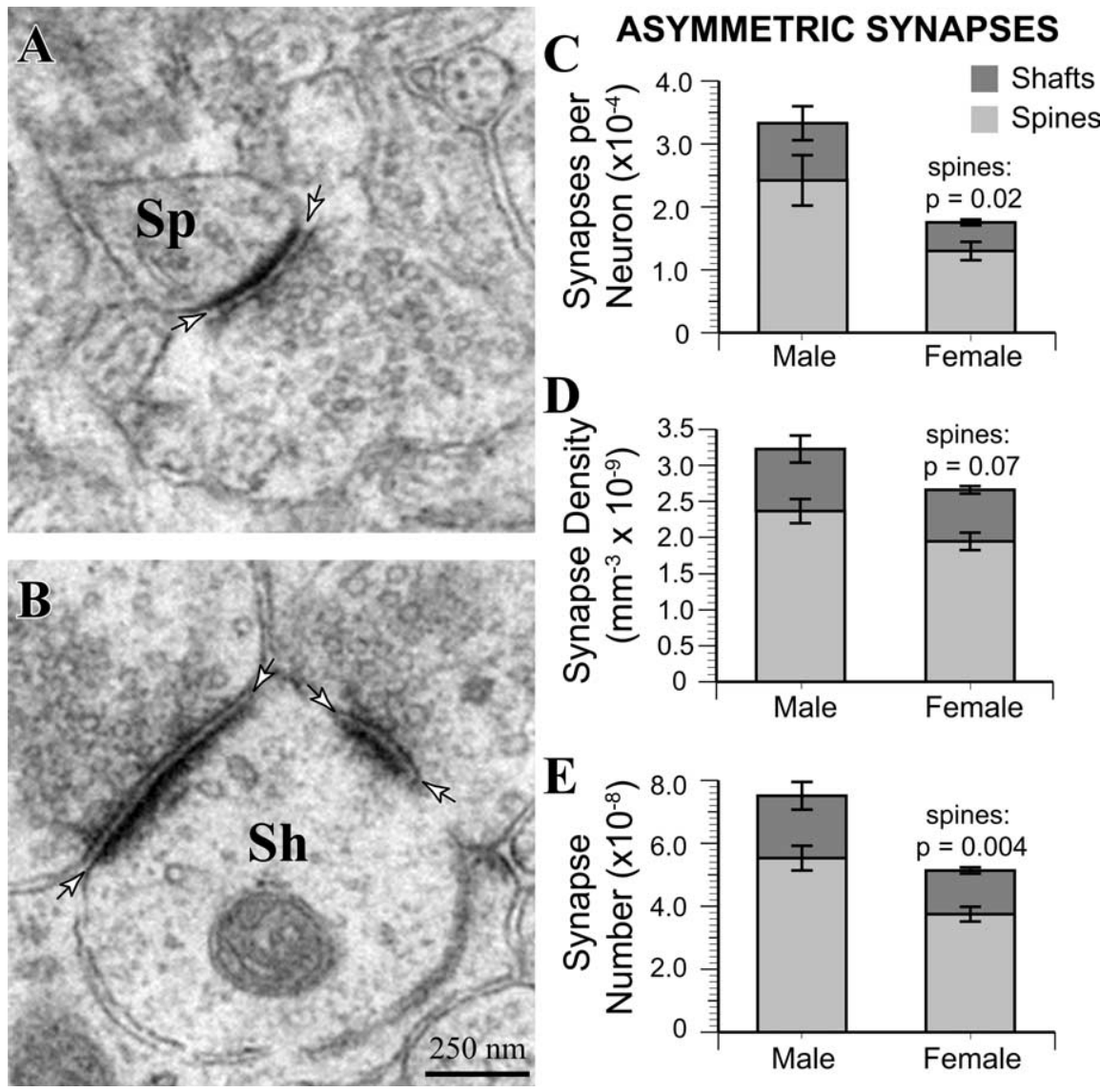

Figure 6. Male MeApd neurons are contacted by more asymmetric spine synapses. $A$, Example of an MeApd asymmetric spine synapse. $\boldsymbol{B}$, Example of MeApd asymmetric shaft synapses. $\boldsymbol{C}$, Asymmetric synapse-to-neuron ratio is greater in males $(p=0.04)$; male neurons have significantly more dendritic spine synapses $(p=0.02)$. $\boldsymbol{D}$, The overall density of asymmetric synapses was statistically equivalent between males and females ( $p=0.1$ ), but males showed a trend toward greater density of asymmetric spine synapses than females ( $p=0.07)$. $\boldsymbol{E}$, The total number of asymmetric synapses was significantly greater in males than in females ( $p=0.02$ ). Furthermore, the total number of asymmetric spine synapses was also significantly greater $(p=0.004)$ in males, whereas the number of asymmetric shaft synapses was statistically equivalent ( $p=0.1$ ). Sh, Shaft synapse; Sp, spine synapse. Arrows indicate boundary of synaptic clefts.

lesser extent than in adults. Thus, our results indicate that previously observed steroid-dependent plasticity in the adult MeA occurs within the context of synapses already organized into male and female phenotypes.

\section{Possible sites of gonadal steroid action}

Our data indicate that MeApd neurons in males receive more excitatory synapses than in females, particularly on dendritic spines. Perinatal exposure to gonadal steroids is involved in the development of most sex differences (Cooke et al., 1998), and the formation of excitatory connections likely reflects coordinated presynaptic and postsynaptic mechanisms ( $\mathrm{Ziv}$ and Garner, 2001). As such, there are at least three possible targets of gonadal steroids that could be involved in producing a greater number of excitatory synapses in the male MeApd: MeApd neurons themselves, intrinsic glutamatergic neurons, and/or glutamatergic afferents.

Neurons in the MeA express both androgen receptors and estrogen receptors (Simerly et al., 1990; Shughrue et al., 1997). Estrogen reportedly increases synaptogenesis in MeA explants grown in the eye of ovariectomized rats (Nishizuka and Arai, 1982). Thus, gonadal steroids could increase the number of asymmetric synapses by acting directly on MeApd neurons to increase their dendritic extent and/or expression of excitatory postsynaptic components, such as postsynaptic density- 95 . Consistent with this idea, overexpression of postsynaptic density-95 increases the number of excitatory synapses on hippocampal neurons in vitro (El-Husseini et al., 2000).

It is also possible that gonadal hormones increase MeApd synapses through their effects on presynaptic neurons. Although $50-90 \%$ of the total population of rat MeA neurons is GABAergic (Mugnaini and Oertel, 1985), glutamatergic neurons have been reported in the gerbil MeApd (Simmons and Yahr, 2003). Because no sex difference was detected in the number of MeApd neurons in the current sample, an effect on intrinsic afferents would likely be accompanied by additional differences; for example, in males, each presynaptic glutamatergic MeApd neuron might form more intrinsic synapses and/or the proportion of glutamatergic MeApd neurons might be greater. Interestingly, the proportion of vasopressinergic neurons in the MeApd is sexually dimorphic (De Vries and Simerly, 2002), providing a precedent for sex differences in the balance of neurochemical phenotypes in this region.

Afferents to the MeApd could also contribute more excitatory synapses in males. The posteromedial cortical nucleus of the amygdala (PMCo) projects to the MeApd (Coolen and Wood, 1998), is not strongly GABAergic (Mugnaini and Oertel, 1985), and is larger in males than in females (Vinader-Caerols et al., 1998). Additionally, mitral cells of the AOB, which are glutamatergic (Quaglino et al., 1999), project directly to the MeApd (Scalia and Winans, 1975) and are more numerous in males than in females (PérezLaso et al., 1997). Thus, the PMCo and the AOB are glutamatergic, sexually dimorphic nuclei that project to the MeApd. If their efferents are more numerous in males, they could contribute more excitatory inputs to MeApd neurons.

\section{MeApd volume is sexually dimorphic before puberty}

An unexpected finding in this study was the sex difference in MeApd volume. Cooke et al. (1999) reported that MeApd volume in castrated adult male rats was equivalent to that in adult females, suggesting that greater MeApd volume in gonadally intact males is dependent on adult circulating androgen. Because hormone levels in the prepubertal rats were not sexually dimorphic, what accounts for this discrepancy? Comparison of MeApd volumes in the present study with those reported for 90-d-old gonadally intact Long-Evans rats (Cooke et al., 1999) indicates that MeApd volume increases more across the two ages in females ( $76 \%$ in females vs $63 \%$ in males). Aside from the possibility of strain differences, this suggests that the female MeApd may grow more during puberty, making the volume of the adult female 
MeApd equal to that in the male in the absence of the activational effects of androgens.

There are several possible sources of the regional volume sex difference observed in our study. Although volumetric sex differences are often caused by differences in cell number (Cooke et al., 1998), we found no evidence of this. Thus, the volume occupied by neuropil, including dendrites, axons, and glia, is likely to be sexually dimorphic. This idea is consistent with the trend toward lower neuronal density that we observed in males. Male MeApd neurons at this age may have larger dendritic arbors, which could provide a substrate for a greater excitatory synapse-to-neuron ratio.

Laterality of MeApd sexual dimorphism The discovery that the functional number of excitatory synapses is sexually dimorphic in the left but not the right MeApd adds to a growing list of reports that the amygdala is lateralized. For example, Cooke et al. (2003) reported greater morphological changes in the left MeApd than the right in response to hormonal manipulations. Why might the MeA be lateralized in these ways? One possibility is that the left MeA is specialized for chemosensory and/or steroid regulation of the secretion of luteinizing hormone $(\mathrm{LH})$. When male rats are exposed to urine from an estrous female, LH levels are increased within several minutes (Kamel et al., 1977). Electrical stimulation of the MeA is known to increase LH secretion (Beltramino and Taleisnik, 1978), and the MeA is well positioned anatomically to mediate signals from the AOB to the neurons that regulate LH release (De Vries and Simerly, 2002). Evidence in support of left-sided control of LH comes from a study of women with temporal lobe epilepsy. Women with left hemisphere foci have abnormally high levels of LH more often than women with foci on the right (Herzog et al., 2003). In addition to chemosensory signals, gonadal steroids also regulate LH levels. LH stimulates gonadal steroidogenesis, and gonadal steroids negatively regulate $\mathrm{LH}$. The MeA has been suggested to play a role in this negative feedback mechanism (Kostarczyk, 1986). Thus, if the left MeA plays a greater role in LH regulation, greater steroid sensitivity may confer a dominant role to the left MeA in the negative feedback regulation of $\mathrm{LH}$ as well.

How MeA laterality is established is unknown; however, of the two studies that have addressed lateralized gonadal steroid receptor expression in rats, both found that estrogen receptor (Sandhu et al., 1986) and androgen receptor (Xiao and Jordan, 2002) expression were greater in the left cortex and hippocampus, respectively. Thus, if the synaptic sex difference reported here is mediated by the activation of gonadal steroid receptors, asymmetry in receptor expression may contribute to the development of sexdependent laterality.

The presence of a lateralized sex difference in the number of excitatory synapses raises the following question: how do the "extra" synapses in males influence the function of the MeA? Recent studies in mice indicate that the vomeronasal organ and the AOB are important in sex and strain discrimination (Stowers et al., 2002; Luo et al., 2003). This suggests that the MeA, which receives input directly from the $\mathrm{AOB}$, may be involved in forming higher-order representations from olfactory stimuli, such as conspecific identity. This idea is supported by the finding that MeA lesions prevent males from avoiding individual conspecifics that defeated them in a recent fight (Luiten et al., 1985) and that oxytocin delivered to the MeA in oxytocin knock-out mice rescues their deficit in social recognition (Ferguson et al., 2001). Although speculative, MeA laterality may be interpreted in light of the theory (Ivry and Robertson, 1998) that the left hemisphere is specialized for processing fine spatiotemporal detail, whereas the right hemisphere dominates the processing of the holistic and global aspects of a stimulus. If this notion is applicable to rats, the greater number of excitatory synapses in the left MeA of males may aid them in male-typical behaviors that require fine sensory discrimination and motor control, such as in male-male competition, detecting estrous females, and modulating $\mathrm{LH}$ release.

\section{References}

Aron C (1979) Mechanisms of control of the reproductive function by olfactory stimuli in female mammals. Physiol Rev 59:229-284.

Baulieu EE, Kelly PA (1990) Hormones. Paris: Hermann.

Beltramino C, Taleisnik S (1978) Facilitatory and inhibitory effects of elec- 
trochemical stimulation of the amygdala on the release of luteinizing hormone. Brain Res 144:95-107.

Bergvall AH, Vega Matuszczyk J, Dahlof LG, Hansen S (1991) Peripheral anosmia attenuates female-enhanced aggression in male rats. Physiol Behav 50:33-40.

Brennan PA, Keverne EB (2004) Something in the air? New insights into mammalian pheromones. Curr Biol 14:R81-R89.

Cooke B, Hegstrom CD, Villeneuve LS, Breedlove SM (1998) Sexual differentiation of the vertebrate brain: principles and mechanisms. Front Neuroendocrinol 19:323-362.

Cooke BM, Tabibnia G, Breedlove SM (1999) A brain sexual dimorphism controlled by adult circulating androgens. Proc Natl Acad Sci USA 96:7538-7540.

Cooke BM, Breedlove SM, Jordan CJ (2003) Both estrogen receptors and androgen receptors contribute to testosterone-induced changes in the morphology of the medial amygdala and sexual arousal in male rats. Horm Behav 43:336-346.

Coolen LM, Wood RI (1998) Bidirectional connections of the medial amygdaloid nucleus in the Syrian hamster brain: simultaneous anterograde and retrograde tract tracing. J Comp Neurol 399:189-209.

De Vries GJ, Simerly RB (2002) Anatomy, development, and function of sexually dimorphic neural circuits in the mammalian brain. In: Hormones, brain and behavior (Pfaff DW, Arnold AP, Etgen AM, Fahrbach SE, Rubin RT, eds), pp 137-191. San Diego: Elsevier.

De Vries GJ, Buijs RM, Van Leeuwen FW (1984) Sex differences in vasopressin and other neurotransmitter systems in the brain. Prog Brain Res 61:185-203.

Dobrunz LE, Stevens CF (1997) Heterogeneity of release probability, facilitation, and depletion at central synapses. Neuron 18:995-1008.

El-Husseini AE, Schnell E, Chetkovich DM, Nicoll RA, Bredt DS (2000) PSD-95 involvement in maturation of excitatory synapses. Science 290:1364-1368.

Ferguson JN, Aldag JM, Insel TR, Young LJ (2001) Oxytocin in the medial amygdala is essential for social recognition in the mouse. J Neurosci 21:8278-8285.

Gerendai I, Halász B (1997) Neuroendocrine asymmetry. Front Neuroendocrinol 18:354-381.

Guillamón A, Segovia S (1997) Sex differences in the vomeronasal system. Brain Res Bull 44:377-382.

Harris VS, Sachs BD (1975) Copulatory behavior in male rats following amygdaloid lesions. Brain Res 86:514-518.

Herzog AG, Coleman AE, Jacobs AR, Klein P, Friedman MN, Drislane FW, Ransil BJ, Schomer DL (2003) Interictal EEG discharges, reproductive hormones, and menstrual disorders in epilepsy. Ann Neurol 54:625-637.

Hines M, Allen LS, Gorski RA (1992) Sex differences in subregions of the medial nucleus of the amygdala and the bed nucleus of the stria terminalis in the rat. Brain Res 579:321-326.

Hsia AY, Malenka RC, Nicoll RA (1998) Development of excitatory circuitry in the hippocampus. J Neurophysiol 79:2013-2024.

Ivry RC, Robertson LC (1998) The two sides of perception. Cambridge, MA: MIT.

Kamel F, Wright WW, Mock EJ, Frankel AI (1977) The influence of mating and related stimuli on plasma levels of luteinizing hormone, follicle stimulating hormone, prolactin, and testosterone in the male rat. Endocrinology 101:421-429.

Kostarczyk EM (1986) The amygdala and male reproductive functions. I. Anatomical and endocrine bases. Neurosci Biobehav Rev 10:67-77.

Luiten PG, Koolhaas JM, de Boer S, Koopmans SJ (1985) The corticomedial amygdala in the central nervous system organization of agonistic behavior. Brain Res 332:283-297.

Luo M, Fee MS, Katz LC (2003) Encoding pheromonal signals in the accessory olfactory bulb of behaving mice. Science 299:1196-1201.
Meaney MJ, McEwen BS (1986) Testosterone implants into the amygdala during the neonatal period masculinize the social play of juvenile female rats. Brain Res 398:324-328.

Meaney MJ, Dodge AM, Beatty WW (1981) Sex dependent effects of amygdaloid lesions on the social play of prepubertal rats. Physiol Behav 26:467-472.

Mouton PR (2002) The principles and practices of unbiased stereology: an introduction for bioscientists. Baltimore: The Johns Hopkins University.

Mugnaini E, Oertel WH (1985) An atlas of the distribution of GABAergic neurons and terminals in the rat CNS as revealed by GAD immunohistochemistry. In: Handbook of chemical neuroanatomy (Björklund A, Hokfelt T, eds), pp 436-622. New York: Elsevier.

Nishizuka M, Arai Y (1981) Sexual dimorphism in synaptic organization in the amygdala and its dependence on neonatal hormone environment. Brain Res 212:31-38.

Nishizuka M, Arai Y (1982) Synapse formation in response to estrogen in the medial amygdala developing in the eye. Proc Natl Acad Sci USA 79:7024-7026.

Pérez-Laso C, Segovia S, Collado P, Rodríguez-Zafra M, Del Abril A, Guillamon A (1997) Estradiol masculinizes the number of accessory olfactory bulb mitral cells in the rat. Brain Res Bull 42:227-230.

Peters A, Palay SL, Webster H (1991) The fine structure of the nervous system, Ed 3. New York: Oxford UP.

Quaglino E, Giustetto M, Panzanelli P, Cantino D, Fasolo A, Sassoe-Pognetto $M$ (1999) Immunocytochemical localization of glutamate and gammaaminobutyric acid in the accessory olfactory bulb of the rat. J Comp Neurol 408:61-72.

Regehr WG, Stevens CF (2001) Physiology of synaptic transmission and short-term synaptic plasticity. In: Synapses (Cowan WM, Sudof TC, Stevens CF, eds), p 767. Baltimore: The Johns Hopkins University.

Sandhu S, Cook P, Diamond MC (1986) Rat cerebral cortical estrogen receptors: male-female, right-left. Exp Neurol 92:186-196.

Scalia F, Winans SS (1975) The differential projections of the olfactory bulb and accessory olfactory bulb in mammals. J Comp Neurol 161:31-55.

Sheehan T, Paul M, Amaral E, Numan MJ, Numan M (2001) Evidence that the medial amygdala projects to the anterior/ventromedial hypothalamic nuclei to inhibit maternal behavior in rats. Neuroscience 106:341-356.

Shughrue PJ, Lane MV, Merchenthaler I (1997) Comparative distribution of estrogen receptor- $\alpha$ and $-\beta$ mRNA in the rat central nervous system. J Comp Neurol 388:507-525.

Simerly RB, Chang C, Muramatsu M, Swanson LW (1990) Distribution of androgen and estrogen receptor mRNA-containing cells in the rat brain: an in situ hybridization study. J Comp Neurol 294:76-95.

Simmons DA, Yahr P (2003) GABA and glutamate in mating-activated cells in the preoptic area and medial amygdala of male gerbils. J Comp Neurol 459:290-300.

Smith ER, Stefanick ML, Clark JT, Davidson JM (1992) Hormones and sexual behavior in relationship to aging in male rats. Horm Behav 26:110-135.

Stowers L, Holy TE, Meister M, Dulac C, Koentges G (2002) Loss of sex discrimination and male-male aggression in mice deficient for TRP2. Science 295:1493-1500.

Vinader-Caerols C, Collado P, Segovia S, Guillamon A (1998) Sex differences in the posteromedial cortical nucleus of the amygdala in the rat. NeuroReport 9:2653-2656.

Xiao L, Jordan CL (2002) Sex differences, laterality, and hormonal regulation of androgen receptor immunoreactivity in rat hippocampus. Horm Behav 42:327-336.

Ziv NE, Garner CC (2001) Principles of glutamatergic synapse formation: seeing the forest for the trees. Curr Opin Neurobiol 11:536-543.

Zucker RS (1989) Short-term synaptic plasticity. Annu Rev Neurosci 12:13-31. 